\title{
Impact of COVID Pandemic on the Children with Cerebral Palsy
}

\author{
Atul R. Bhaskar ${ }^{1} \cdot$ Mayuri V. Gad ${ }^{2}(1) \cdot$ Chasanal M. Rathod $^{3}$ \\ Received: 30 September 2021 / Accepted: 7 December 2021 / Published online: 18 January 2022 \\ (c) Indian Orthopaedics Association 2022
}

\begin{abstract}
Background The COVID pandemic has been raging across the world for the past 18 months and has severely impacted healthcare and resources. Children with special needs have been adversely affected by the COVID pandemic, due to lack of formal schooling, access to rehabilitation and limited physical and social activity. The aim of this online survey was to understand the effect of the pandemic on the children with Cerebral Palsy (CP).

Methods An online survey was conducted using a nine parts questionnaire comprising 26 questions. Survey included questions pertaining to demographic data, ambulatory status, lack of access to physiotherapy, orthotic adjustment, surgery, deterioration of function, and behaviour. A simple binary answer was sought to cover all social strata of society.

Results Responses were received from 101 caregivers who participated in an online questionnaire. $25.7 \%$ had no therapy sessions, $74.2 \%$ were on therapy of which $23.7 \%$ had online sessions. The lockdown restrictions coupled with lack of rehabilitation and orthotic support led to deterioration in physical function in 54 children and worsening of deformity in 34 . Changes in behavioural pattern was observed in 45 children.

Conclusion The survey revealed major disruptions in the care of Children with CP. The parents reported difficulties in managing the child therapy at home. There is always a possibility that the pandemic will result in a lockdown again, and hence our approach toward physical therapy assessment and rehabilitation needs to be towards home-based and family-centred care.
\end{abstract}

Keywords Cerebral palsy $\cdot$ COVID pandemic $\cdot$ Orthosis $\cdot$ Rehabilitation $\cdot$ Behavioural pattern

\section{Introduction}

The SARS COVID-19 pandemic with recurrent waves of infection has severely impacted the healthcare system globally. While the adults have borne the brunt of infection, children have suffered its indirect consequences. In March 2020 , more than 652,473 cases of coronavirus disease 2019 (COVID-19) were reported in India due to which a sudden and complete lockdown was enforced in April 202 [1].

Mayuri V. Gad

mayurigad123@gmail.com

Atul R. Bhaskar

atul25670@gmail.com

Chasanal M. Rathod

chasanal@gmail.com

1 Department of Orthopaedics, Children Orthopaedic Clinic, Andheri, Mumbai, India

2 St. Xavier's Gait Lab, Mahim, Mumbai, India

3 Department of Orthopaedics, SRCC Children's Hospital, Haji Ali, Mumbai, India
Maharashtra state accounted for approximately $22.35 \%$ of the total cases in India.

Childhood disability is a huge burden in every society. About one in every six children within the age group of 2-8 years worldwide have some form of neurodevelopmental, behavioural, or emotional disorder [2]. These children with special needs [autism, attention deficit hyperactivity disorder, CP, learning disability, developmental delays, and other behavioural and emotional difficulties] and their parents have encountered numerous challenges during the current pandemic and lockdown [2]. The sudden interruption of schools, access to rehabilitation, and medical treatment have led to new challenges for caregivers. Depending on severity, these children have a routine for various therapies such as physiotherapy, occupational therapy, speech therapy, psychomotor therapy, and orthotic visits. According to Wang $\mathrm{G}$ et.al, children with $\mathrm{CP}$ consult a physician about 5 times a year and attend almost four therapy sessions per week [3]. Another survey highlighted the negative effects of the lockdown on the children's wellbeing and their mental and social health (morale, behaviour, social interaction, 
and physical activity), similar to a recent report on children without disability [4]. The psychological effects of the lockdown, including post-traumatic stress symptoms, confusion, and anger have been reported in the general population and are related to the duration of the lockdown [5-7]. Children with physical disabilities have an increased risk of mental health symptoms, which could be exacerbated during the pandemic [7-10].

The aim of this study was to understand the effect of the COVID Pandemic in children with CP through an online survey as regards accessibility to physical therapy, procurement of orthosis/splints, follow up visits with specialty doctors (Neurologist, Orthopaedic Surgeon, and others), hip surveillance and individual behavioural changes in children with CP.

\section{Materials and Methods}

\section{Participants}

The study was conducted during the COVID-19 pandemic in the period between March 2020 and March 2021. The study population consisted of children diagnosed with CP. Parents of children with $\mathrm{CP}$ were contacted by email or messaging app. A Google survey form link was sent to all participants. The study population included children with a diagnosis of CP that were under the care of two Paediatric Orthopaedic Surgeons. All these children were attending supervised physiotherapy sessions at various rehabilitation centres and maintained their scheduled visits with their specialty doctors until the pandemic struck.

\section{Development of the Survey}

The survey was developed by using the online google survey forms. The Survey was developed in English and Hindi language. The survey consisted of 9 parts with 26 questions. The first part was related to the introduction and demographic data about the child. The second part of the survey was related to online therapy and home program. The questions were related to regular therapy, online session feedback, and parents performing therapy at home. The third part was about the requirement in orthosis and splints and feedback on accessibility to modify or procure a new orthosis/splint. The fourth part included follow-up visits with specialty doctors (Neurologist, Orthopaedic Surgeon, and others), hip surveillance. The fifth and sixth part was focused on actual physical status, ambulatory potential, and any loss of function. The seventh part was about any delay in intervention. The eight-part aims on assessing behavioural changes in children during the lockdown. The ninth part is comprised of general information on COVID-19 infection in the family. Most questions had closed answers, either binary (yes/no) or multiple choice.

\section{Statistical Analysis}

Descriptive analysis was performed on the 101 responses that were fulfilled. Quantitative results are expressed as mean (standard deviation) and categorical results as number $(\%)$.

\section{Results}

Caregivers of 101 children with $\mathrm{CP}$ with a mean age of 10.3 years (range 2-18 years) responded to the questionnaire and were included in the study. There were 66 boys and 35 girls in the study. As per the topographic classification of $\mathrm{CP}$, the majority of children were diplegics (60.3\%) followed by hemiplegics $(24.7 \%)$, triplegic $(7.92 \%)$ and quadriplegic were (6.93\%). The ambulatory status was rated according to the Gross Motor Functional Classification System (GMFCS) grade. The ambulatory CP children i.e., Level I, II, III were $90 \%$, and non-ambulatory Level IV and V were 9.09\% (Table 1).

The second part of the survey assessed the disruption of physiotherapy services. 39 (38.6\%) children were on regular therapy sessions, 36 (35.6\%) children were on intermittent therapy sessions and 26 (25.7\%) children did not undergo any therapy session in the lockdown period due to lack of access to a therapist. Out of the 39 children who were on regular therapy, 13 (12.87\%) parents opted for a home visit by the therapist, 17 (16.83\%) children took the home program, $8(7.92 \%)$ children took the combination therapy

Table 1 Demographic data of children with CP

\begin{tabular}{lll}
\hline Children with CP & Mean (SD) & Range \\
\hline Age & $10.32(5.27)$ & $2-18$ \\
Gender & $n$ & $\%$ \\
Male & 66 & 66.3 \\
Female & 35 & 33.7 \\
Type of CP & $n$ & $\%$ \\
Diplegie & 61 & 60.3 \\
Hemiplegie & 25 & 24.7 \\
Triplegie & 8 & 7.92 \\
Quadriplegie & 7 & 6.93 \\
GMFCS & $n$ & $\%$ \\
Level I & 26 & 25.74 \\
Level II & 37 & 36.6 \\
Level III & 28 & 27.72 \\
Level IV & 9 & 8.91 \\
Level V & 1 & 0.99 \\
\hline
\end{tabular}


(home visit by a therapist, home program, online therapy with a therapist) and only one child took online therapy. For children who were on an intermittent therapy session, 11 (10.89\%) children underwent home visit by the therapist, $10(9.90 \%)$ children took the home program, 10 (9.90\%) children underwent combination therapy (home visit by a therapist, home program, online therapy with a therapist) and only five children took online therapy. A total of 24 children took online therapy, out of which seven parents (30\%) were satisfied with the session. The parents also expressed their concern regarding their inability and difficulty in the home program. Around 60 (59.4\%) parents tried to perform regular exercises at home, 30 (29.7) parents were unable to perform therapy due to lack of knowledge, and 11 (10.9\%) parents did not perform any exercise for their child (Fig. 1).

Regarding the orthotic support (i.e., 3rd part of the questionnaire), many parents did experience growth spurt in the children and the need for change/modification of orthosis or splints. $51(50.5 \%)$ parents expressed the need to make new AFO or splints and could not physically reach out or visit the orthotist as the vendor's shops were closed due to strict lockdown rules.
Specialist consultation in terms of personal face to face visits at the clinic/hospital or online consultation were almost similar in number. Out of 101 children 49 (48.51\%) children opted for hospital or clinic visits, while 51 (50.49\%) children had online consultation/follow-up. Among 49 children who had opted for hospital or clinic visits, 27 (26.73\%) children had orthopaedic consultations, 7 (6.9\%) children had neurological consultation and 15 (14.9\%) children had a consultation with other doctors (paediatrician, ophthalmologist, etc.). In the online consultation group 24 (23.76\%) children had orthopaedic consultation, 22 (21.8\%) children had neurological consultation and $5(5 \%)$ children had a consultation with any other doctors(paediatrician, ophthalmologist, etc.) (Fig. 2).

The COVID restrictions also impacted the physical function of the child. Any deterioration of physical function in the child and the need for surgical intervention was assessed in the 5th to 7th part of the questionnaire. Due to unavailability of rehabilitation services and inability of the parent to perform home exercises, there was noticeable deterioration in the ambulatory status in $57(56.4 \%)$ children. Thirtyfour $(33.7 \%)$ children showed worsening in the function of

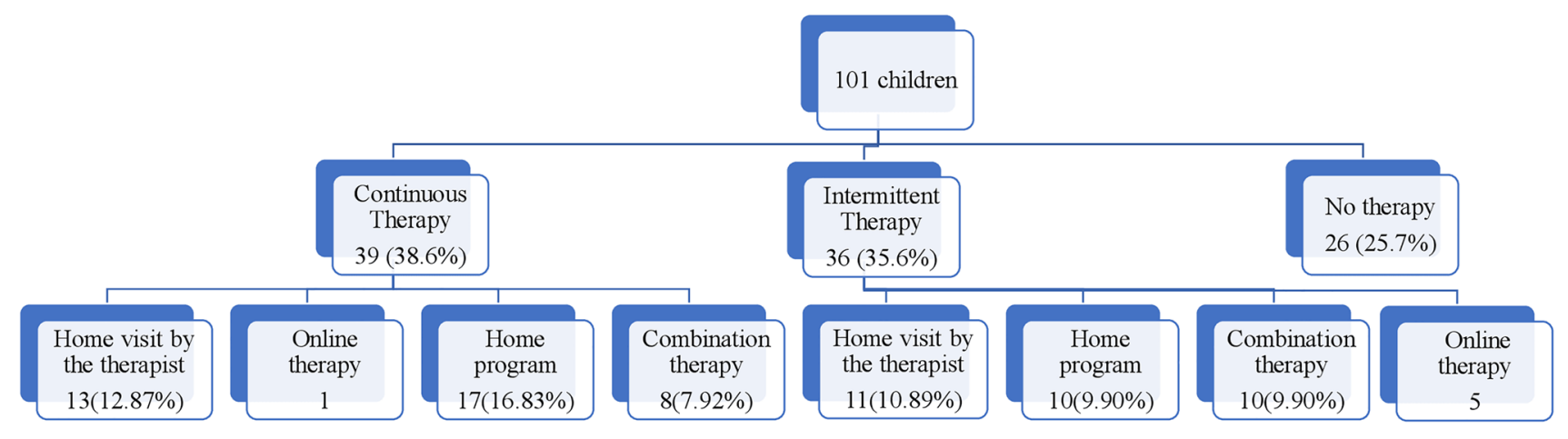

Fig. 1 Rehabilitation sessions during COVID pandemic

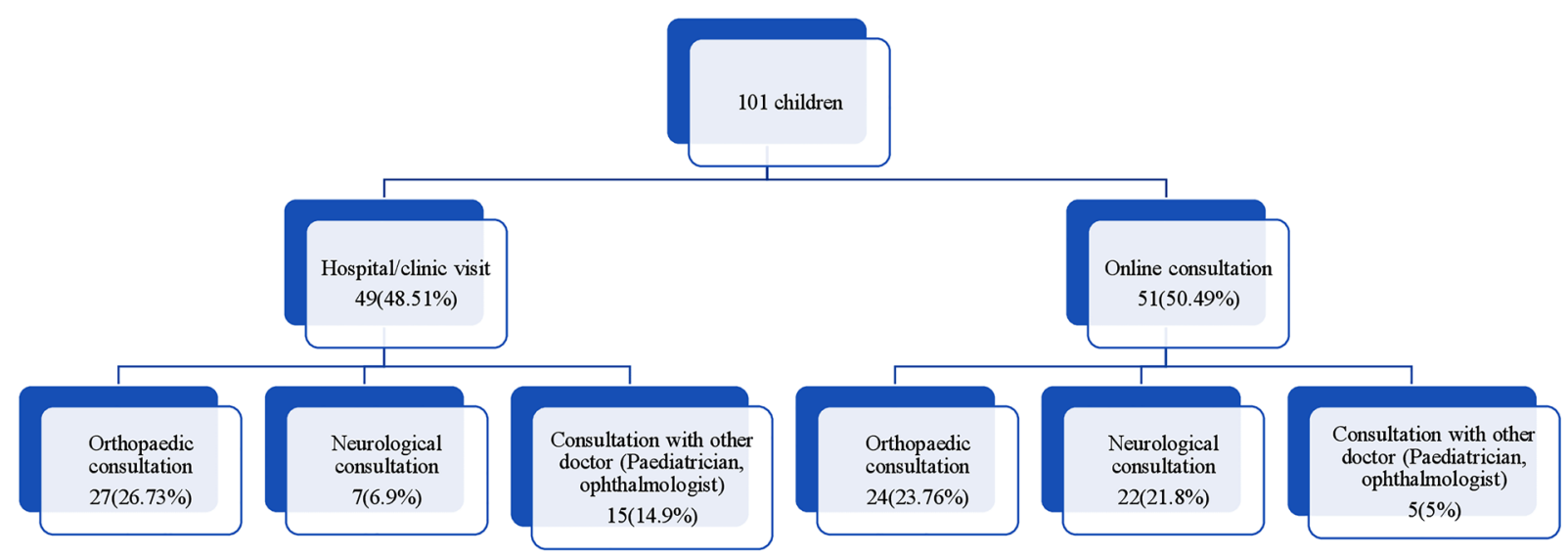

Fig. 2 Speciality doctor consultation and follow up during COVID pandemic 
the upper limb. 42 (41.6\%) parents reported that children had increased joint contracture and were stiffer due to the deformity. 64 (63.4\%) children had an increase in muscle stiffness or tightness as reported by parents (Fig. 3). Surgical intervention was advised in $49(48.5 \%)$ children before the pandemic and lockdown; however, only 29 (28.7\%) children could undergo surgery.

The last part (8th and 9th section of the questionnaire) assessed the behavioural changes as reported by parents. Altered behavioural pattern was seen in 45 (44.6\%) children due to lockdown at home. This was expressed as an increase in irritability, anger issues, and even aggression in $46(45.5 \%)$ children. A significant change in the eating pattern of $29(28.7 \%)$ children was noticed. Weight gain was noted in $30(29.7 \%)$ due to the increased sitting time and sedentary behaviour. 21 (20.8\%) families had suffered COVID-19 infection, but only eight children had their therapy impacted due to morbidity/mortality in the family.

\section{Discussion}

The results of our study showed that the caregiver among the $\mathrm{CP}$ children was largely affected by the COVID- 19 pandemic in terms of deterioration of function, progression of deformities, inability to access regular physical therapy/orthotic services, and developed behavioural issues. A small group of caregivers benefited from teleconsultation and telerehabilitation; parents exhibited their incompetence and difficulty to follow the home program as advised by the therapist. There have been few reports in the literature that discuss the impact of the COVID-19 pandemic on children and caregivers with special needs in terms of rehabilitation/telerehabilitation and mental health [11].

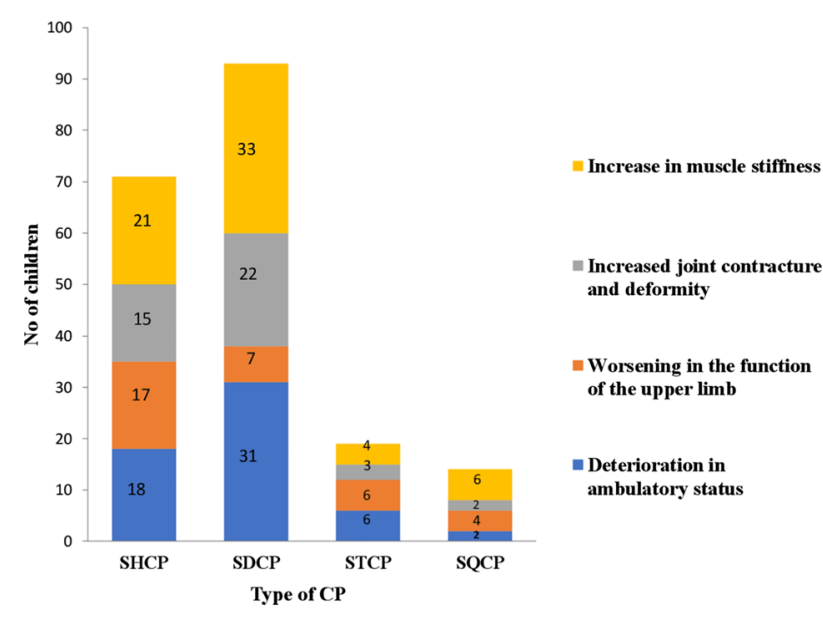

Fig. 3 Deterioration in function of upper limb and lower limb in different type of $\mathrm{CP}$
In our study, $25.7 \%$ of children discontinued therapy due to the lockdown, while a large percentage (35.6\%) of the group had combination therapy (home visit by a therapist, home program, online therapy with a therapist). The caregivers reported only $30 \%$ satisfaction with tele-rehabilitation therapy sessions. However, irrespective of tele-sessions, $60 \%$ of parents tried to perform regular exercises at home. A study from Italy suggested only $5.9 \%$ of 68 children continued conventional physiotherapy; 19 (27.9\%) of 68 children discontinued their conventional program, and telerehabilitation and indirect remote supervision were used in 23.5\% (16/68) and 42.6\% (29/68) of cases, respectively [12]. A study from Turkey showed 12 children (12.8\%) discontinued their physical therapy sessions during the pandemic [13]. In order to prevent the risk of infection and to maintain adherence to government laws in the lockdown a higher number of families discontinued the therapy sessions. A large number of hospitals, therapy centres tried to provide teleservices during the lockdown. Although the caregivers in our study were not very comfortable following the home program as suggested on the online sessions and were less compliant. Telemedicine cannot completely substitute face-to-face clinics. However, it is most beneficial, where conventional healthcare is lacking or impossible to provide accessible, continuous care, with real-time visualization [14]. During the stay home period children had growth in height and weight (girth) which lead to the need for change in splints or orthoses. We found that $50.5 \%$ of parents express their need for making new orthoses or splints during peak lockdown. A study for Iceland, studied about use of orthoses during pandemic, they found that $30.9 \%$ children could not continue use of orthoses [13]. This should be considered as an important point during the pandemic situation as it helps in some extension in recurrence of deformity.

One of the main results from our survey was that $56.4 \%$ of children had experienced deterioration in ambulatory status. $33.7 \%$ children showed worsening in the function of the upper limb, $41.6 \%$ of parents reported that children had increased joint contracture, and $63.4 \%$ of children had an increase in muscle stiffness or tightness. A study by Cankurtaran et.al found that $45.7 \%$ of children had a general health issue, $55.4 \%$ of children had reduced mobility, $58.5 \%$ of children had increased spasticity, and $61.7 \%$ of children had decreased joint motion during the pandemic [13]. Our results showed a significant change in ambulatory change in $53.46 \%$ of children, an increase in muscle tone $56.43 \%$ of children, $38.61 \%$ of children had a change in deformity, and $29.70 \%$ of children had decreased hand function in the affected hand. Ambulatory children (GMFCS levels I, II, and III) had increased muscle tone, decreased upper extremity function, and deterioration in deformity when compared to non-ambulatory children. In non-ambulatory children (GMFCS level IV and V) ambulatory change was seen in 


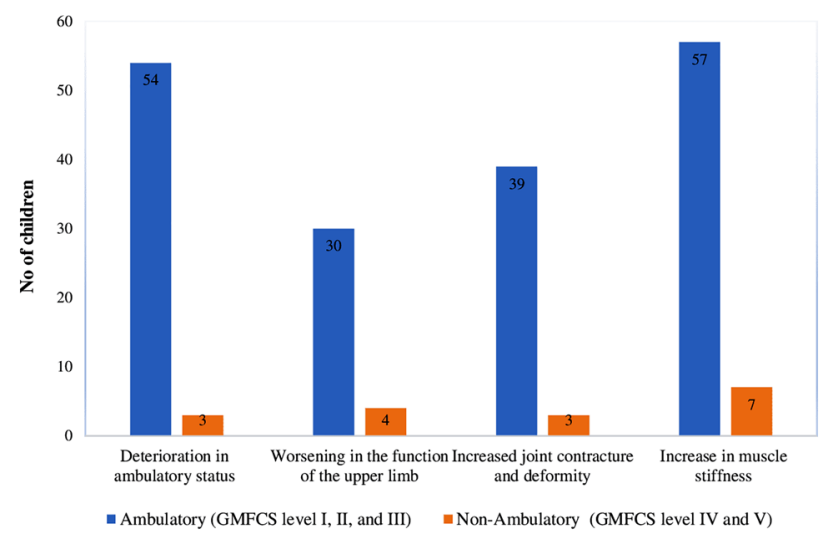

Fig. 4 Deterioration in function of upper limb and lower limb in Ambulatory (GMFCS I, II, III) and Non Ambulatory (GMFCS IV, V)

three children, increased muscle tone in seven children, contracture and progression of deformity was reported in three children, and in four children had worsening of hand function in the involved extremity (Fig. 4). A similar study by Kübra Seyhan Biy1k et.al reported that $75 \%$ of children had upper extremity impairment, and $73 \%$ of children had lower extremity impairment. They found that they increase muscle tone(adductor muscles, hamstrings) and restricted joints in non-walking children (GMFCS level IV and V) than walking children (GMFCS level I, II, and III) [15].

The negative effects of lockdown on children's mental health, wellbeing, and social health (morale, behavior, social interaction, and physical activity) were highlighted in a French survey study [15]. 44.6\% of children reported behavior changing in stay home periods. This was expressed as an increase in irritability, anger issues, and even aggression in $46(45.5 \%)$ children. French study concluded that in total, $55 \%$ of children showed behavior changes, mostly behavioral problems (32\%) and sleeping difficulties (22\%) [15]. A study reported social function and communication $(51.1 \%)$, and mood (55.4\%) were worse during the pandemic compared before [13]. Another study stated that symptoms of depression, anxiety, and stress were found in $62.6 \%, 20.5 \%$, and $36.4 \%$ of caregivers of children with special needs in India during the COVID-19 pandemic, respectively [16]. In our study, we also found a change in the eating pattern of $28.7 \%$ of children. Due to home confinement children could not go to school or participate in outdoor activity resulting in weight gain. $29.7 \%$ reported increase in weight gain greater than $5 \mathrm{~kg}$ impacting physical activity.

\section{Conclusion}

The lockdown in response to the COVID-19 pandemic has disturbed the daily routine for children with $\mathrm{CP}$ and even their family. Changes in life routines, physical activity and participation level affect changes of sensation level of pain, muscle tone, joint movements, stress and sleep duration of children with $\mathrm{CP}$. The survey revealed major consequences such as therapy services were massively interrupted, the parents had difficulties in managing the child therapy at home. There is always a possibility that a 3rd wave in the pandemic will repeat itself, so our approach towards physical therapy assessment and rehabilitation of children with disabilities needs to be re-evaluated and revamped by shifting our focus toward home-based and family-centred care. These approaches can help bridge the gap in the delivery of physical therapy services for children with disabilities in times of COVID-19. The study has also unlocked the future potential of online therapy and care which may be a blessing in disguise for GMFCS 4 and 5 patients with caregiver challenges such as working parents or single parents in nuclear families.

\section{Limitations}

This study had few limitations. This is a pilot study with a small number of participants. Socio-economic strata of the population or parents analysed was not analysed, this could have given a different aspect to reduced therapy session. No physical examination was possible to document the actual progression of deformity. We could not compare pre COVID upper extremity and lower extremity deformities and had to rely on data provided by the caregivers.

Acknowledgements We would like to thank all the experts in the focus group and parents for participating in our study.

\section{Declarations}

Conflict of interest On behalf of all authors, the corresponding author states that there is no conflict of interest.

Ethical Approval This article does not contain any studies with human or animal subjects performed by the any of the authors.

Informed Consent For this type of study informed consent is not required.

\section{References}

1. Goel, I., Sharma, S., \& Kashiramka, S. (2021). Effects of the COVID-19 pandemic in India: An analysis of policy and technological interventions. Health Policy and Technolog, 10, 151-164.

2. Sacaze, E., Garlantezec, R., Rémy-néris, O., Peudenier, S., Rauscent, H., le Tallec, H., Bernier-Francois, V., Pichancourt, D., Brochard, S., et al. (2013). A survey of medical and paramedical involvement in children with cerebral palsy in Britanny: Preliminary results. Annals of Physical and Rehabilitation Medicine., 56(4), 253-267.

3. Wang, G., Zhang, Y., Zhao, J., Zhang, J., \& Jiang, F. (2020). Mitigate the effects of home confinement on children during the COVID-19 outbreak. The Lancet., 395(10228), 945-947. 
4. Brooks, S. K., Webster, R. K., Smith, L. E., Woodland, L., Wessely, S., Greenberg, N., \& Rubin, G. J. (2020). The psychological impact of quarantine and how to reduce it: Rapid review of the evidence. The Lancet., 395(10227), 912-920.

5. Lee, J. (2020). Mental health effects of school closures during COVID-19. The Lancet Child \&amp; Adolescent Health., 4(6), 421.

6. Zhou, S., Zhang, L., Wang, L., Guo, Z., Wang, J., Guo, Z., Wang, J., Chen, J., Liu, M., Chen, X., \& Chen, J. (2020). Prevalence and socio-demographic correlates of psychological health problems in Chinese adolescents during the outbreak of COVID-19. European Child and Adolescent Psychiatry, 29(6), 749-758.

7. Sigurdardottir, S., Indredavik, M. S., Eiriksdottir, A., Einarsdottir, K., Gudmundsson, H. S., \& Vik, T. (2010). Behavioural and emotional symptoms of preschool children with cerebral palsy: A population-based study. Developmental Medicine \&amp; Child Neurology, 52(11), 1056-1061.

8. Steele, M., Taylor, E., Young, C., McGrath, P., Lyttle, B. D., \& Davidson, B. (2008). Mental health of children and adolescents with Duchenne muscular dystrophy. Developmental Medicine \&amp; Child Neurology, 50(8), 638-639.

9. Klein, B. (2016). Mental health problems in children with neuromotor disabilities. Paediatrics \&amp; Child Health, 21(2), 93-96.

10. Keeney, T. (2020). Physical therapy in the COVID-19 pandemic: Forging a paradigm shift for rehabilitation in acute care. Physical Therapy, 100(8), 1265-1267.

11. BanninkMbazzi, F., Nalugya, R., Kawesa, E., Nimusiima, C., King, R., Van Hove, G., \& Seeley, J. (2021). The impact of COVID-19 measures on children with disabilities and their families in Uganda. Disability \&amp; Society. https://doi.org/10.1080/ 09687599.2020.1867075
12. Bertamino, M., Cornaglia, S., Zanetti, A., Di Rocco, A., Ronchetti, A., Signa, S., Severino, M., Moretti, P., Gaslini Stroke Study Group. (2020). Impact on rehabilitation programs during COVID-19 containment for children with pediatric and perinatal stroke. European Journal of Physical and Rehabilitation Medicine, 56(5), 692-694.

13. Cankurtaran, D., Tezel, N., Yildiz, S. Y., Celik, G., \& Unlu, A. E. (2021). Evaluation of the effects of the COVID-19 pandemic on children with cerebral palsy, caregivers' quality of life, and caregivers' fear of COVID-19 with telemedicine. Irish Journal of Medical Science, 190(4), 1473-1480.

14. Ben-Pazi, H., Beni-Adani, L., \& Lamdan, R. (2020). Accelerating telemedicine for cerebral palsy during the COVID-19 pandemic and beyond. Frontiers in Neurology, 26(11), 746.

15. Bıyık, K. S., Özal, C., Tunçdemir, M., Üneş, S., Delioğlu, K., \& Günel, M. K. (2021). The functional health status of children with cerebral palsy during the COVID-19 pandemic stay-at-home period: A parental perspective. The Turkish Journal of Pediatrics, 63(2), 223-236.

16. Dhiman, S., Sahu, P. K., Reed, W. R., Ganesh, G. S., Goyal, R. K., \& Jain, S. (2020). Impact of COVID-19 outbreak on mental health and perceived strain among caregivers tending children with special needs. Research in Developmental Disabilities., 107, 103790.

Publisher's Note Springer Nature remains neutral with regard to jurisdictional claims in published maps and institutional affiliations. 